Article DOI: https://doi.org/10.35219/ann-ugal-math-phys-mec.2019.1.06

\title{
VIBRATION OF RECTANGULAR PLATES: FUNDAMENTAL MODE AND INTEGER MULTIPLE OF THE FUNDAMENTAL PERIOD OF VIBRATION
}

\author{
Maria (Stan) Necula ${ }^{1,2}$, Dorin Bibicu ${ }^{3,4,5}$, Luminita Moraru ${ }^{1,5 *}$ \\ 1 Faculty of Sciences, Physics Department, "Dunărea de Jos" University of Galati, Romania, e-mail: \\ luminita.moraru@ugal.ro \\ 2 Technical College "Radu Negru" Galati, Romania, marcost95@yahoo.com \\ 3 Faculty of Business Administration, 'Dunărea de Jos' University of Galați, Romania, Administration \\ Department, dorin.bibicu@ugal.ro \\ 4 High School "Dunărea," Romania; bibicud@yahoo.com \\ 5 University "Dunărea de Jos" of Galați, Romania, Modelling \& Simulation Laboratory.
}

\begin{abstract}
This paper is concerned with a vibration analysis of the transverse vibrations of rectangular plates with freelysupported boundaries and corners fixed, in an imagistic way, to describe the deformation state for the specific natural frequency and mode shape for each degree-of-freedom. Two materials, i.e. aluminum and steel, showing the homogeneous properties in the direction of plate thickness were chosen. The exact solutions for free vibrations of the homogeneous rectangular plate were obtained and the fundamental mode and integer multiple of the fundamental period of vibration were provided. The comparisons show that the analytical model predicts natural frequencies reasonably well for a rectangular plate.
\end{abstract}

Keywords: imagistic way, free vibration, FEM analysis

\section{INTRODUCTION}

The vibrational analysis has increased during the last three decades, particularly in the aerospace and civil engineering. Plate vibration solutions available in an imagistic way are necessary to describe the deformation state for the specific natural frequency and mode shape for each degree-of-freedom. Various studies on the free vibration of rectangular plates with a wide range of support conditions were reported [1-4]. Also, solution for forced vibration analysis are proposed in [5].

The finite element method FEM is currently used to describe the free vibration of plates by hypothesizing a displacement field model that satisfies the convergence criteria. The FEM allows to determine the natural frequencies of a rectangular plate. Ahmadian and Zangeneh [6] proposed a numerical technique based on FEM and the concept of super elements to analyzed the rectangular plate vibration problems. There are several practical models with a sound mathematical formulation able to describe physical behavior of a vibrating plate with freely-supported boundaries and corners fixed. For fixed corners plates many models have been reported. Gharaibeh and Obeidat [7] reported the solution for a thin elastic rectangular, isotropic and orthotropic, plates with fixed corners using the 
Rayleigh method. Onwuka et al. [8] analyzed the vibration of an all-round-clamped rectangular thin orthotropic

plate, by using Taylor- Mclaurin shape function and Ritz method. They reported that the fixed corners plates have natural frequencies up to $2 \%$ higher than simply supported plates.

The goal of this study is to determine the natural frequencies computed for two shift frequencies, for aluminium and steel rectangular plates with freely-supported boundaries and corners fixed. The percentage differences between the natural frequencies computed for four mode shapes show that the analytical model predicts natural frequencies reasonably well for a rectangular plate.

\section{MATERIALS AND METHOD}

A thin plate is defined as having a small thickness in comparison to the planer dimensions and the analysis becomes a 2D problem. When a force is applied on normal direction to the surface of the plate, it bends in two directions and a twisting moment appears.

In the framework of this study, the materials are assumed to be isotropic, homogeneous with density $\rho$, Young's modulus E and Poisson's ratio v. The Kirchhoff's plate theory is used. Let w denote the deflection of the surface of the plate in the $\mathrm{z}$ direction. The total strain energy of the plate in terms of the displacement $\mathrm{w}(\mathrm{x}, \mathrm{y})$ is $[1]$ :

$U=\frac{D}{2} \int_{0}^{b} \int_{0}^{a}\left[\left(\frac{\partial^{2} w}{\partial x^{2}}\right)^{2}+\left(\frac{\partial^{2} w}{\partial y^{2}}\right)^{2}+2 \mu\left(\frac{\partial^{2} w}{\partial x^{2}}\right)\left(\frac{\partial^{2}}{\partial y^{2}}\right)+2(1-\mu)\left(\frac{\partial^{2} w}{\partial x \partial y}\right)^{2}\right] d x d y$

$T_{p}=\frac{1}{2} \rho h \int_{0}^{a} \int_{0}^{b} w^{2} d x d y$

And the kinetic energy

where the plate stiffness factor is, $\mathrm{D}=\frac{E h^{3}}{12\left(1-\mu^{2}\right)}$

E=elastic modulus

$\mathrm{h}=$ plate thickness

$\mu=$ Poisson's ratio

$\mathrm{X}$-Y plane is located in the plate surface (i.e. $\mathrm{z}=0$ ). The in-plane contributions of the kinetic energies is neglected. The variation of the displacement components $U$ and $V$ across the thickness are as follows:

$$
\begin{gathered}
\mathrm{U}=-\mathrm{Z} \frac{\partial w}{\partial w} \quad \varepsilon_{x}=\frac{\partial u}{\partial x}=-Z \frac{\partial^{2} w}{\partial x^{2}}= \\
\varepsilon_{y}=\frac{\partial u}{\partial y}=-Z \frac{\partial^{2} w}{\partial x y^{2}} \\
\Upsilon_{x y}=\frac{\partial u}{\partial y}+\frac{\partial v}{\partial x}=-2 z \frac{\partial^{2} w}{\partial x \partial y}=
\end{gathered}
$$

The direct strain is expressed as and the shear strain as

$$
\left\{\begin{array}{c}
\varepsilon_{x} \\
\varepsilon_{y} \\
\Upsilon_{x y}
\end{array}\right\}=-Z\left[\begin{array}{c}
\frac{\partial^{2}}{\partial x^{2}} \\
\frac{\partial^{2}}{\partial y^{2}} \\
\frac{\partial^{2}}{\partial x \partial y}
\end{array}\right]
$$


The matrix form of these equations is .

The transverse displacement of a vibrating plate equation is given by

$$
D\left[\frac{\partial^{4} w}{\partial x^{4}}+2 \frac{\partial^{4} w}{\partial x^{2} \partial y^{2}}+\frac{\partial^{4} w}{\partial y^{4}}\right]+\rho \frac{\partial^{z} w}{\partial t^{2}}=0
$$

$t$ is time and $\rho$ is the mass per unit area of the plate.

For fixed corners plates, the Rayleigh's method, which is based on the energy conservation theorem is used.

\section{RESULTS AND DISCUSSION}

The same thin plate (size $\mathrm{x}=8$ inch, $\mathrm{y}=12$ inch, thickness of 0.5 inch) is discretized into a $4 \times 6$ grid and analyzed using the FEM and the corresponding frequencies are obtained. The transverse vibrations of this rectangular plates with freely-supported boundaries and corners fixed and the mode shape are simulated. Two materials, i.e. aluminum and steel, showing the homogeneous properties in the direction of plate thickness are used. Figure 1 shows the grid used in FEM analysis.

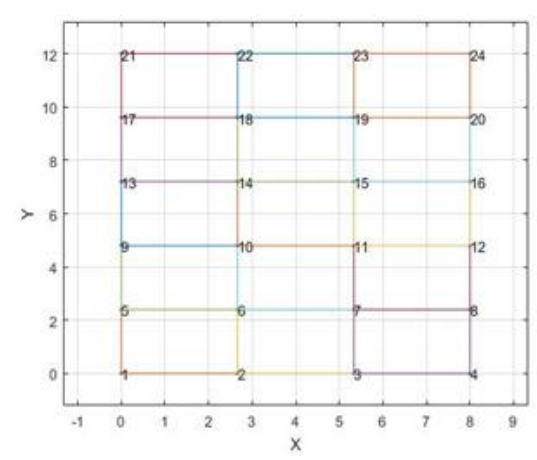

Fig.1. Finite plate of size $x=8$ inch, $y=12$ inch, thickness of 0.5 inch. The plate is divided into a 4 x 6 grid. The shift frequencies: $25 \mathrm{~Hz}$ and $100 \mathrm{~Hz}$.

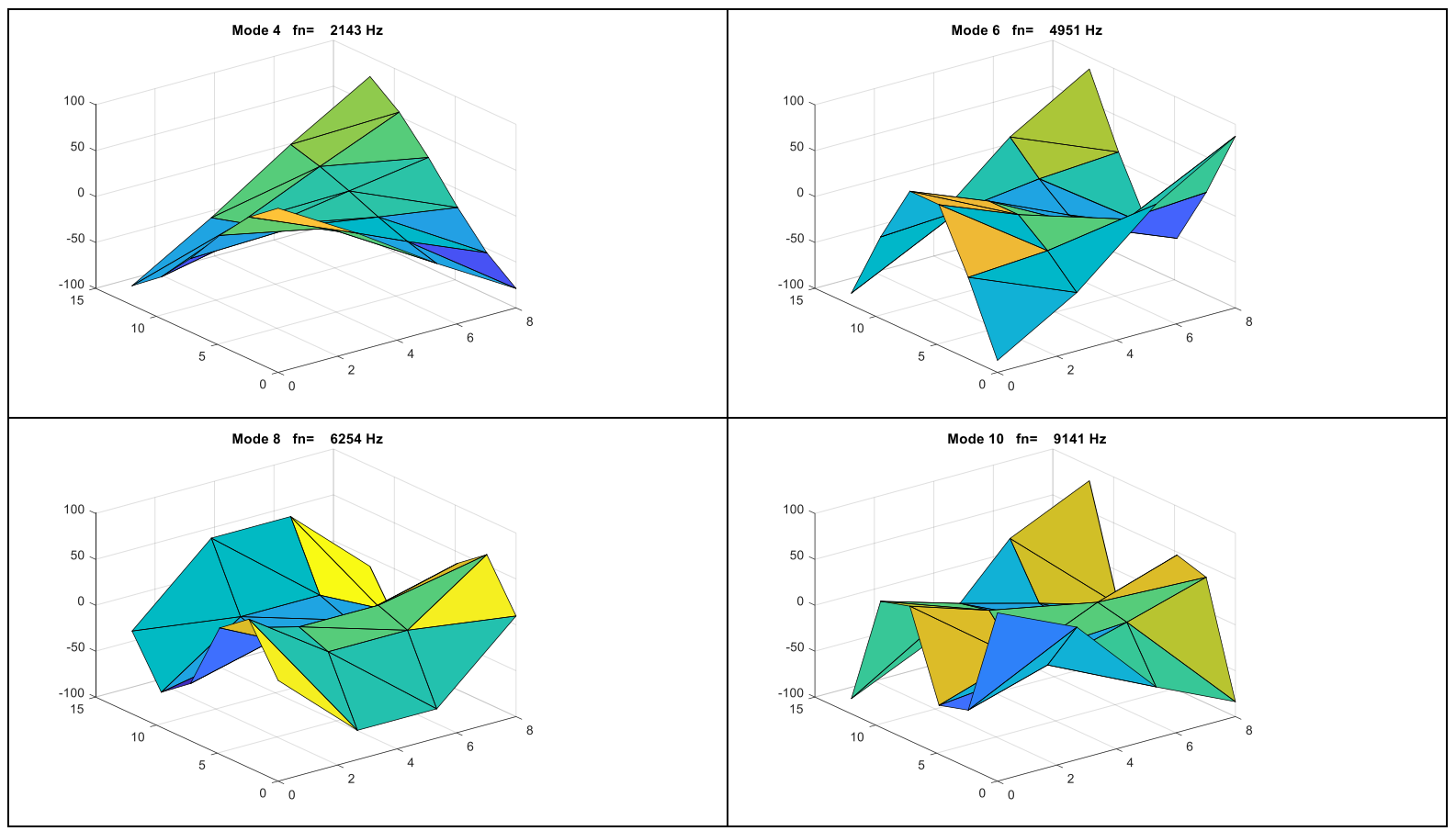

a) 


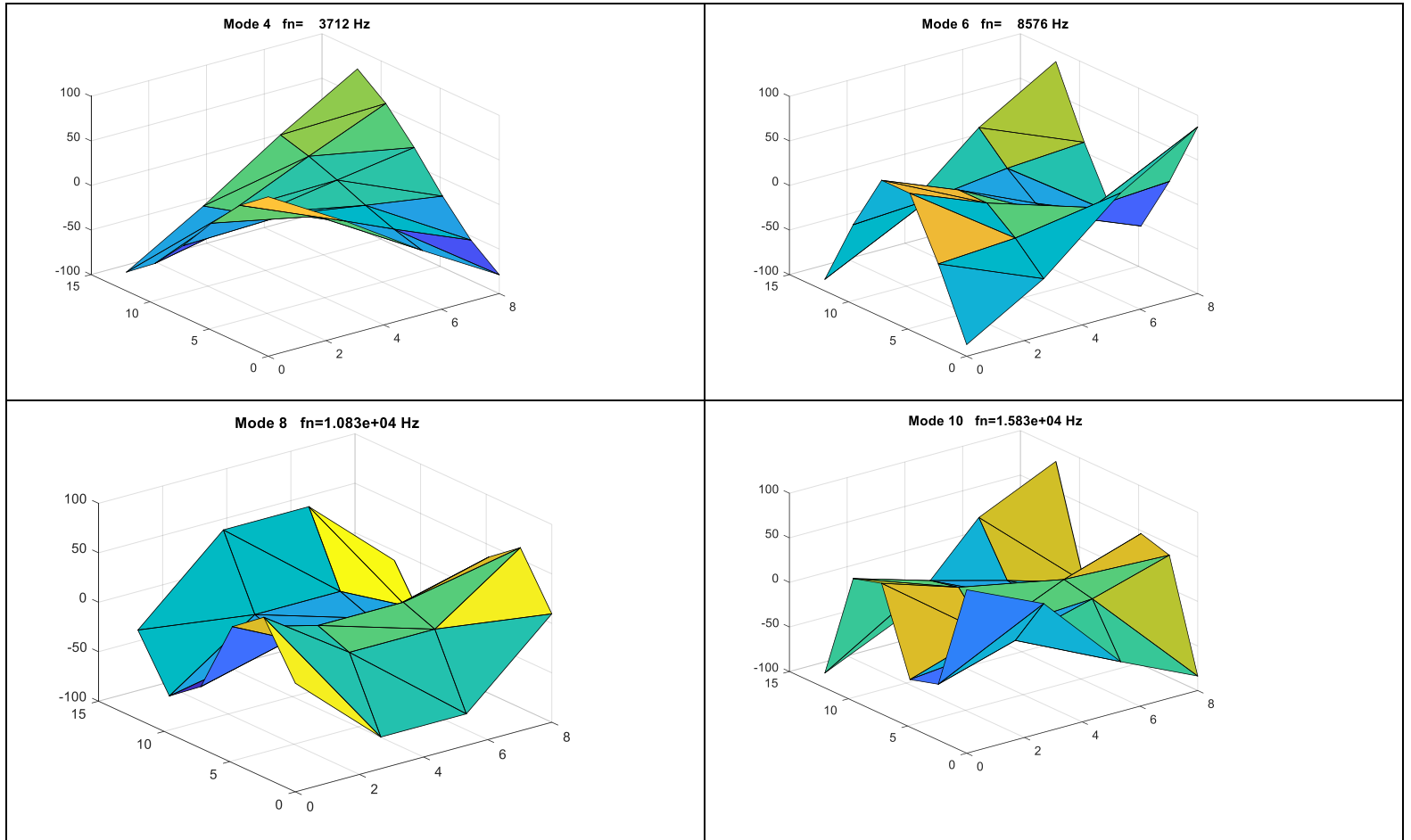

b)

Fig. 2. Mode of vibration for a freely-supported homogeneous plate. A) aluminium; b) steel. The shift frequency $25 \mathrm{~Hz}$, thickness $0.5 \mathrm{inch}$, mass $0.5 \mathrm{lbm}$, boundary conditions: all free

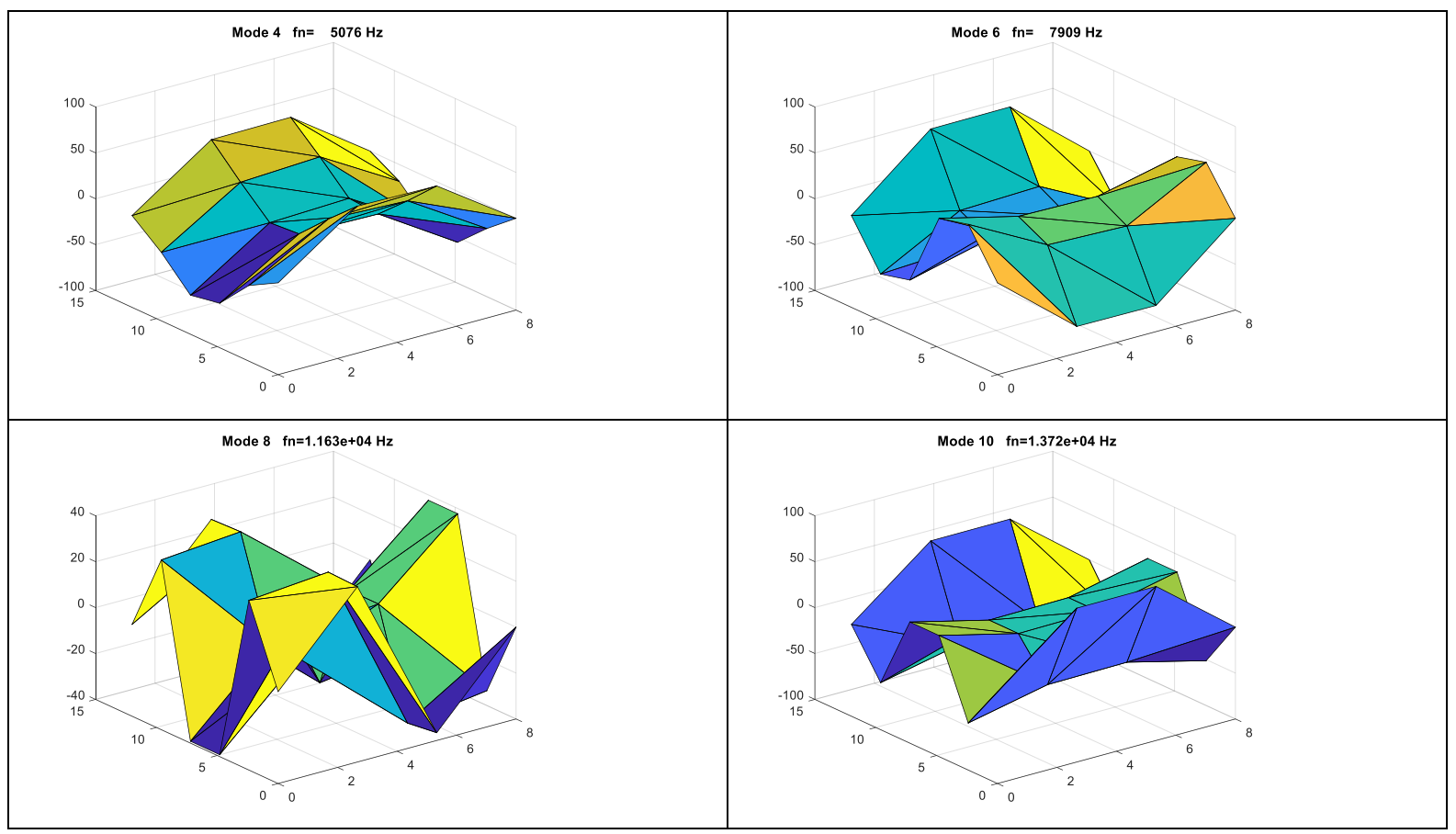

a) 


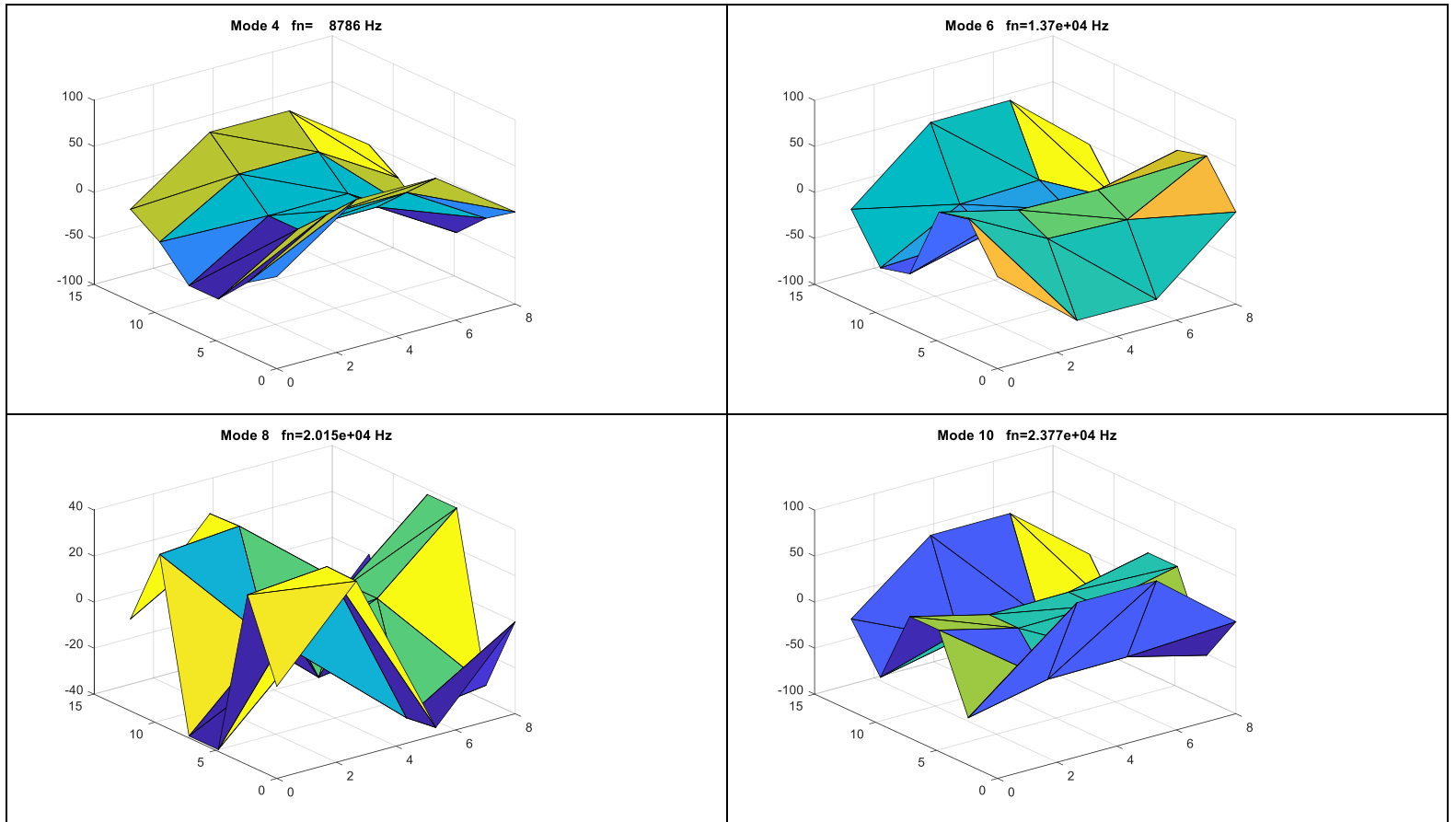

b)

Fig. 3. Mode of vibration for a homogeneous plate with corners fixed. A) aluminium; b) steel. The shift frequency $25 \mathrm{~Hz}$, thickness $0.5 \mathrm{inch}$, mass $0.5 \mathrm{lbm}$, boundary conditions: corners fixed

Figures 2 and 3 display two examples of the shape mode of vibration for freely-supported and corners fixed homogeneous plates. Only four modes are analyzed to save the computation time.

Tables 1 and 2 give the corresponding natural frequencies for all experimental conditions.

The highest frequency values were computed for fixed corners and steel. So, it is clear indicated that once the boundary condition changes from free supported to fixed corners, the frequencies increase. Also, the frequencies increase starting to mode 4 towards mode 10.

Table 1. Natural frequencies for aluminium and steel; shift frequency $25 \mathrm{~Hz}$

\begin{tabular}{|c|c|c|c|c|c|}
\hline \multirow{3}{*}{ Mode } & \multicolumn{5}{|c|}{ Natural frequencies $\vartheta(\mathrm{Hz})$ FEM calculation } \\
\hline & \multicolumn{2}{|c|}{ boundary all free } & \multirow[t]{2}{*}{ Mode } & \multicolumn{2}{|c|}{ corners fixed } \\
\hline & $\vartheta_{A \mathbb{P}}^{\text {fves }}$ & $\theta_{\text {Stgel }}^{\text {fNeg }}$ & & $\vartheta_{A P}^{f I X E d}$ & $\vartheta_{\text {Stgeg? }}^{\text {fixed }}$ \\
\hline 4 & 2142.8 & 3711.6 & 4 & 5076 & 8786.4 \\
\hline 6 & 4951.2 & 8575.7 & 6 & 7908.9 & 13699 \\
\hline 8 & 6253.5 & 10831 & 8 & 11632 & 20148 \\
\hline 10 & 9141.4 & 15833 & 10 & 13723 & 23768 \\
\hline
\end{tabular}

Table 2. Natural frequencies for aluminium and steel; shift frequency $100 \mathrm{~Hz}$

\begin{tabular}{|c|c|c|c|c|c|}
\hline \multirow{3}{*}{ Mode } & \multicolumn{5}{|c|}{ Natural frequencies $\vartheta(\mathrm{Hz})$ FEM calculation } \\
\hline & \multicolumn{2}{|c|}{ boundary all free } & \multirow{2}{*}{ Mode } & \multicolumn{2}{|c|}{ corners fixed } \\
\hline & $\vartheta 1_{A P}^{\text {fres }}$ & $\vartheta 1_{\text {Stgel }}^{\text {freg }}$ & & $\vartheta 1_{A I}^{\text {fixgd }}$ & $91_{\text {Stgel }}^{\text {fixed }}$ \\
\hline 4 & 2140.6 & 3710.4 & 4 & 5072.8 & 8786.4 \\
\hline 6 & 4950.2 & 8575.2 & 6 & 7908.9 & 13699 \\
\hline 8 & 6252.8 & 10831 & 8 & 11632 & 20148 \\
\hline 10 & 9140.9 & 15833 & 10 & 13723 & 23768 \\
\hline
\end{tabular}


Table 3. The percentage differences between the natural frequencies obtained for shift frequencies of 25 and $100 \mathrm{~Hz}$

\begin{tabular}{|c|c|c|c|c|c|}
\hline \multirow{3}{*}{ Mode } & \multicolumn{5}{|c|}{ The percentage differences } \\
\hline & \multicolumn{2}{|c|}{ boundary all free } & \multirow[t]{2}{*}{ Mode } & \multicolumn{2}{|c|}{ corners fixed } \\
\hline & 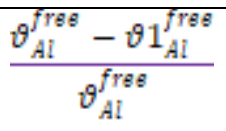 & $\frac{\vartheta_{\text {Steal }}^{\text {freg }}-\vartheta 1_{\text {Stagl }}^{\text {freg }}}{\vartheta_{\text {Stael }}^{\text {frag }}}$ & & $\frac{\vartheta_{A I}^{f \text { fixgd }}-\vartheta 1_{A I}^{f \text { Lxgd }}}{\vartheta_{A I}^{\text {fuxgd }}}$ & $\frac{\vartheta_{\text {Stgal }}^{f \text { fugdl }}-\vartheta 1_{\text {Stgei }}^{\text {fuxed }}}{\vartheta_{\text {Steal }}^{\text {fixed }}}$ \\
\hline 4 & 0.102 & 0.03 & 4 & 0.06 & 0 \\
\hline 6 & 0.020 & $5,83 \mathrm{E}-05$ & 6 & 0 & 0 \\
\hline 8 & 0.011 & 0 & 8 & 0 & 0 \\
\hline 10 & $5,46 \mathrm{E}-05$ & 0 & 10 & 0 & 0 \\
\hline
\end{tabular}

Table 3 shows that both results tend to converge as the shape mode increase. For aluminium, the results diverge for lower shape modes. Similar comparison indicates a perfect convergence for steel.

\section{CONCLUSIONS}

In this study, boundary condition effect, i.e. freely-supported and corners fixed, on free vibration behavior of homogeneous plates is investigated. Frequency values are compared for 4 vibration modes. Also, two shift frequencies were studied. When the shape mode increases the influence of shift frequency is diminished. The effect is more pronounced for fixed corners plate.

\section{References}

1. Yuemei Liu, Rui Li, Accurate bending analysis of rectangular plates with two adjacent edges free and the others clamped or simply supported based on new symplectic approach, Applied Mathematical Modelling 34, pp 856-865, 2010.

2. Li, R., Zhong, Y., Tian, B., Liu, Y. M., On the finite integral transform method for exact bending solutions of fully clamped orthotropic rectangular thin plates. Applied Mathematics Letters., Vol. 22 (12), pp. 1821-1827, 2009.

3. Leissa A.W., "Vibration of Plates", NASA SP-160, Washington DC, 1969.

4. Leissa A.W., "Plate vibration research 1976-1980: classical theory", Shock vibration Digest, 13, pp.11-12, 1981.

5. Sakata T., Hosokawa K., "Vibrations of analysis of clamped orthotropic rectangular plates", Sound Vibr., 125(3), pp.429-439, 1988. 